\title{
Advancing Attention Control Using VR-Induced Multimodal Artificial Synesthesia
}

\author{
John H. Reif ${ }^{1,2}$ \\ ${ }^{1}$ Department of Computer Science, Duke University, \\ Durham, NC 27707 USA and \\ ${ }^{2}$ Adjunct Faculty of Computing and Inf. Tech. (FCIT), \\ King Abdulaziz University (KAU), Jeddah, Kingdom of \\ Saudi Arabia
}

\author{
Wadee Alhalabi ${ }^{2,3}$ \\ ${ }^{2}$ Faculty of Computing and Inf. Tech. (FCIT), \\ King Abdulaziz University (KAU), \\ Jeddah, Kingdom of Saudi Arabia \\ ${ }^{3}$ Virtual Reality Research Center, Effat University,
}

\begin{abstract}
This paper is an interdisciplinary study of novel applications of techniques and tools of an area of brain science, known as Synesthesia (involving associations and/or confusion between distinct senses), to area of Computer Science known as Immersive Virtual Reality (VR), that makes the subject's awareness of physical self be diminished by being surrounded in an engrossing artificial environment. Natural Synesthesia has for the last decade been an important emerging area in brain science but is present in only a small proportion of the population. For example a person with Natural Synesthesia, when viewing a grapheme, may perceive a color additionally to be associated to the grapheme. In contrast, Artificial synesthesia (also known as virtual synesthesia or synthetic synesthesia) has been defined as the sensory joining due a cross-modal mapping device, where information of one sense is accompanied by an induced perception in another sense. In particular, we propose use of a multimodal manner of displaying information in VR to increase and concentrate attention.

Artificial Synesthesia to synthetically create induced associations between senses, allowing Artificial Synesthesia to be experienced by anyone using a VR system. The paper describes the enhancement of immersive VR by use of Artificial Synesthesia to improve the system's performance at steering and directing the attention of the user. We describe techniques for an enhanced immersive VR that displays associations between a variety of senses: between colors and characters, also between colors and sounds, and between sounds and the position of tactile sensations. The sense association provided by Artificial Synesthesia allows the system to better capture the user's attention and better direct that attention. A major application of our work in VR-induced Artificial Synesthesia is to provide an enhanced methodology for controlling the attention of the subject, and to improve the direction of attention of subjects undergoing guided imagery therapies for pain relief. Other potential high-impact applications include improved immersive VR, more programmable human/computer interfaces and other medical therapies.
\end{abstract}

Keywords - virtual reality (VR); immersive; synesthesia; synesthaesia; artificial synesthesia; pain therapy 


\section{INTRODUCTION}

\section{I.1 Virtual Reality}

Virtual Reality (VR) is an important area of computer science that generates computer-simulated environments that simulate physical presence of a subject; these simulate various sorts of physical presence that may include both visual, as well as additional sensory information, such as sound or tactile sensations. Immersive VR is VR that makes the subject's awareness of physical self be diminished by being surrounded in an engrossing artificial environment provided by the VR system.

\section{I.2 Prior Application of Immersive VR for Pain Therapy}

Immersive VR has been quite successful in relieving the pain of patients by diverting the attention of the patient away from the pain. Enhanced methods for diverting the attention of patients have an immediate application to improved pain therapies.

\section{I.3 The Challenge}

A key challenge for Immersive VR, which is a central concern of this paper, is directing the attention of the subject and to enhance and enrich chosen perceptions. (For example, directing the attention of the subject away from pain, or to improve guided imagery therapy directing the attention of the subject to progress the subjects mind-state from an initial mind-state through a series of mind-states. )

\section{I.4 Our Interdisciplinary Approach: Applying Ideas from Brain Science to Immersive VR}

One of the most productive paradigm for doing research in an area of computer science is to take key techniques and ideas from another field, and then apply these to the area of computer science of interest, providing a leap in innovation not otherwise likely or possible. A research strategy that can potentially provide enhanced impact in this or any other topic of research is the introduction of a technology or technique not previously used in the field. Often such a technology comes from another field, and so it was not previously conceived as applicable to the field of research of interest. What might be a potential novel (to conventional practice in the field) applicable technology for the field of VR?

We believe that Brain Science provides some key techniques for Immersive VR. Applying this paradigm, the main thrust of this paper is to enhance Immersive Virtual Reality (VR) by the use of powerful emerging techniques and ideas in Brain Science.

Specific topic of our Paper: The field of brain science is large, and so we have narrowed our paper's discussion to a specific subarea of brain science (a) that has not been much investigated and (b) can be of significant benefit to VR. We feel that Synesthesia is such a topic. In particular, this is an interdisciplinary paper applying in a novel way techniques and tools of an emerging area of brain science known as Synesthesia to Immersive VR.

Our Novel Use of VR Technology: VR appears to be ideal in its ability to create an immersive perceptual experience. The use of immersive VR allows for especially vivid, 3D renderings of associations between distinct pairs or larger groups of distinct senses. To our knowledge, little or no prior research exists in training induced Synesthesia, as defined below.

\section{I.5 Natural Synesthesia}

Natural Synesthesia (also spelled synæsthesia or synaesthesia) denotes the class of neurophysiological phenomena where the subject has made associations between distinct senses. (To avoid confusion, in this paper we preface the term Natural to the term Synesthesia to indicate that the neurophysiological phenomena is not induced by for example VR). In Natural Synesthesia, the sensory associations that the subject perceives appear to be real perceptions, but are created within the brain. A synaesthetic (a person experiencing Natural Synesthesia) does not normally perceive an uncolored grapheme as colored; instead they perceive colors to be associated to the grapheme. These can include for example: perception of particular colors associated with particular graphemes (e.g., visual perceived characters or numbers), sounds, tactile sensations, taste or smells.

A wide variety of types of Natural Synesthesia are well known to exist in a relatively few individuals, and so is present in only a small proportion of the population. Examples of Natural Synesthesia involving color and other senses include:(a) Perception of particular colors associated with particular graphemes (e.g., visual perceived characters or numbers), (b) Perception of particular colors associated with particular sounds, (c) Perception of particular colors associated with tactile sensations, (d) Perception of particular colors associated with taste or smells, and so on. Similar types associations have been demonstrated in certain individuals between nearly all pairs of the types of senses, as well as between groups of more than two types of senses.

Enhanced attention of subjects experiencing Natural Synesthesia: In most cases, the subjects experiencing Natural Synesthesia note that their perception of objects involving Natural Synesthesia are very much enhanced, for example characters or words that are perceived to have associated colors are thus much more noticed in the visual field. Natural Synesthesia is often experimentally verified by testing the increase facility that a subject with Natural Synesthesia can detect 
certain patterns or objects, due to the sensory associations (e.g., colored graphemes) that highlight the objects. Hence Natural Synesthesia provides a unique opportunity for enhancing certain perceptions.

However Natural Synesthesia is present in only a small proportion of the population.

Our work is motivated by some interesting properties of Natural Synesthesia:

(a) It appears in some cases to initially occur early in the maturation process,

(b) It appears to be affected by certain drugs and hormones,

(c) It appears to have a genetic component.

Neural plasticity is a general term for the ability of the Brain to change dynamically. These properties (a)-(c) point to the possibility that Natural Synesthesia might be actually a byproduct of neural plasticity. The enhanced neural plasticity that is known to occur during maturation, appears to sometimes cause Synesthesia. Also the neural plasticity induced by various drugs and hormones can induce Synesthesia.

\section{I.6 Artificial Synesthesia generated by VR: Inducing Synesthesia in Mature Individuals without the use of Drugs or Harmones}

Neural plasticity is now known to occur in fully mature individuals as well, even without use of drugs or hormone. A small number of studies have demonstrated that one can induce Synesthesia via training, perhaps this is due to the subjects' neural plasticity.

Artificial Synesthesia (also known as Synthetic Synesthesia) is associations between senses created synthetically, for example use of VR to display Synesthesia associations between distinct senses. Natural Synesthesia has for the last decade been an important emerging area in brain science; we feel Artificial Synesthesia could have similarly important applications to VR.

While Natural Synesthesia is present in only a small proportion of the population, in contrast Artificial Synesthesia can be potentially experienced by anyone using a VR system.

A few prior demonstrations of Synthetic Synesthesia exist and are described in the section on prior research; this lists a small but emerging body of prior works on the use of Artificial Synesthesia to enhance VR, for example to color particular objects in a visual scene to enhance the subject's attention to those objects. However, these prior works in Artificial Synesthesia in VR have not exploited the underlying structure of the senses involved.

The main thrust of our paper is to enhance immersive VR by use of Artificial Synesthesia. Our paper describes an enhanced Immersive VR that induces, in the subjects' brain, associations between a variety of senses: between colors and characters, also between colors and sounds, and between sounds and the position of tactile sensations. These induced sensory associations appear to be associated with increased attention by the user; hence they potentially provide a method for improving the system's control of the user's attention, to direct the users attention to intended states of the mind (e.g., to states of the mind without pain).

We also exploit the known underlying structure of various senses, as described in the next section.

1.7 The Advantage of Training of Artificial Synesthesia to Enhance Control of Subjects Attention: Once training of Artificial Synesthesia is done, then we conjecture that this training can be exploited to allow for enhanced control of the human subject's attention:

(a)Enhanced Attention Control via Increased Intensity of Perception of a Synesthesia-Grouped set of Senses: We conjecture that if all of a Synesthesia-grouped set of senses are simultaneously experienced by a subject the subject's attention will shift so that the subject will be much more attentive to that Synesthesia-grouped stimuli as compared to the conventional case where only one sensory perception may be used to attempt to control the subject's attention.

(b) Enhanced Attention Control via Increased Attention Directed to other Synesthesia-Grouped set of Senses: We also conjecture that if only one of a given sensations of a Synesthesia-grouped set of senses is initially perceived by a subject, the subject's attention will shift so that the subject will be much more attentive to the other Synesthesia-grouped set of senses that might also be present but not previously perceived by the subject. Hence if the subject only initially notices one of a given Synesthesia-grouped set of senses, then the subject is much more likely to perceive the other sensations of a Synesthesiagrouped set of senses, if they are also present.

Hence the direction of the attention of the subject may be much more effectively controlled by presenting the subject with Synesthesia-grouped set of senses. In layman terms, we conjecture that the Synesthesia-grouping of senses acts like a searchlight to highlight perception and attention by the subject.

\section{I.8 Structured Artificial Synesthesia generated by VR}

Each of the various senses such as color, character visual processing, sound pitch and spatial tactile sensations appear to have an underlying structure, modeled in prior works by a spatial representation (a continuous $2 \mathrm{D}$ maps) which we call a sense-map. For example, (a) colors can be arranged in perceived similarity around a wheel to form a color-map, (b) tones can be arranged in an array (sometimes called call a tone-map) that reflects similarity of frequency as well as tonal patterns of groups of sounds such as chords, and (c) the sensory homunculus is a one-to-one correspondence of an area of the body to a specific position on the brain. (See the section III.4 for more details and paper citations.) 
A further highly innovative goal of our paper is to also exploit the rich underlying structure of the senses. The various senses such as color, character visual processing, sound pitch and spatial tactile sensations each are known to have an underlying complex structure, generally modeled in prior works by a spatial representation (a continuous 2D map) which we term a sense-map (e.g., color maps for visual perception, tone and chord maps for sounds, etc.). We discuss enhancing immersive VR by first known use of what we call Structured Artificial Synesthesia, where the coupling between senses also couples the structure of the various senses by a mapping between the corresponding sense-maps. To do this, we choose mappings between sense-maps of distinct senses (for example transfer of pleasant harmonic chords to pleasantly perceived groupings of another sense such as of colors).

\section{I.9 Potential High-Impact Applications VR-induced Artificial Synesthesia}

The enhanced ability provided by Artificial Synesthesia for controlling the subject's attention, and for detection patterns or objects has many potential applications. Potential applications of VR-induced Artificial Synesthesia include improved immersive VR, more programmable human/computer interfaces and medical therapies. In particular, a major application is for subjects suffering pain; this paper develops improved methods for directing the attention of subjects away from pain. Other potential high-impact applications of this research include improved immersive VR, more programmable human/computer interfaces and other medical therapies.

\section{Use of Virtual Reality (VR) for Pain Therapy}

\section{II.1 Prior Literature on Use of Virtual Reality (VR) for Pain Therapy:}

Pain Distraction Therapy: Relief of severe pain is a major problem in the field of Medicine. Fortunately and according to extensive prior literature, all sorts of pain can be distracted via controlled sensory stimuli. Considerable prior work by the coauthor (e.g. [1]) and many others (e.g., [2], [3]) has demonstrated the use of Virtual Reality (VR) for distracting attention for patients experiencing pain, and thus reducing the pain they experience. This is sometimes known as VR distraction therapy for pain. VR distraction therapy for pain does not completely eliminate pain, but can provide considerable relief from the perception of the degree of pain. The degree of success of distraction therapy depends on the degree the patient is distracted by the sensory stimuli provided by the VR system.

Using Immersive VR for Pain Distraction Therapy: Pain Distraction Therapy is one of the most important applications of Immersive VR in medical applications. [4] have proved that virtual reality can be used as an alternative effective nonpharmacologic analgesia to reduce dental pain and discomfort. Thus it allows patients to tolerate painful dental procedures safely. [5] proved that VR has a huge effect on reducing heart pulse rates during dental procedures, especially in children. [6] studied the use of virtual reality as a distraction tool to connect patients to a virtual environment. With fifty participants undergoing routine gastric laboratory procedures, $86 \%$ of them testified to the effectiveness of the system.

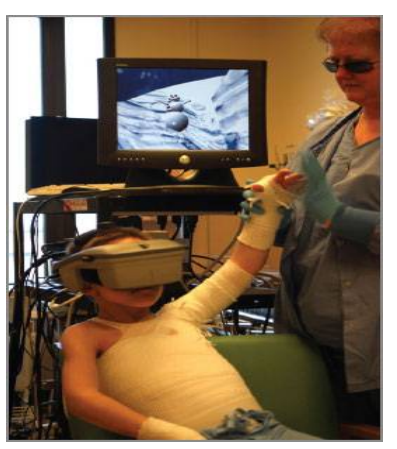

Figure 1A Using Immersive VR for Pain Distraction Therapy (from [2] Hoffman H.G., Patterson D.R., Carrougher G.J., \& Sharar S.(2001). The Effectiveness of Virtual Reality Based Pain Control with Multiple Treatments, Clinical Journal of Pain, 17, 229-235)

We may look at the example provided by Dr Hoffman and Dr Patterson [2] at the University of Washington, as illustrated in Figure 1A. The team created an immersive VR environment called Snow World, which is designed to reduce pain during the burn therapy by distracting attention. To experience Snow World during physical therapy sessions, patients wear a helmet hooked up to the virtual world configured as an icy 3D canyon, where patients can throw snowballs on snowmen, igloos, and penguins, providing physical therapy to stretch the newly healed skin. It was more effective and worked by distracting attention away from pain to the 3D world, decreasing Pain $\mathrm{dx}$ by $40 \%-50 \%$ [2]. In [7] a study was conducted on patients were each patient was treated for three minutes of physiotherapy without diverting their attention. Another three minutes using virtual reality games, and used the visual analog scale to measure the pain intensity. The result shows a reduction in the treatment time from 60 minutes to 14 minutes.

II.1B Pain Reduction by Imagery Therapy using Immersive VR: 
Imagery therapy is a particular type of methods for pain distraction where images are presented to achieve the desigered paid distraction. The co-author Alhalabi and his team have in prior work made considerable contributions to pain reduction via both VR diversion as well in particular imagery therapy to control pain and to help patients of many diseases including rheumatoid arthritis [8], post-stroke paralysis [9], [10], dentist [12], and cancer [13], [14], and pain in general [11]. [15] reported that virtual reality system has been used with imagery therapy to distract patients' attention away from the painful real world to a positive virtual environment which helps in alleviating pain in numerous diseases including leukemia, breast cancer, burns, phobia, and many others. From prior data and scientific evidences, we can infer that the percentage of imagery therapy methods using virtual reality techniques which have been successfully implemented in controlling pain associated with rheumatoid arthritis is very high. It is been demonstrated that distracting attention is critical to success of therapy.

Pain Distraction using Unguided Image Therapy: In some of this Image Therapy work, the patient is provided with controls to freely wonder through the virtual world. As an example, the co-author developed [11] a system using imagery therapy via virtual reality to relieve pain using his system "Arrival Dream". This system immerses patients in the environment, to attract their attention away from the clinic environment to the forest environment. Arrival Dream is introduced to drive burn patients into relaxation and adventure. It allows patients to control the movement using joystick and other input devices. Patients walk between grass and trees, and are exposed to other natural animals and live such as frogs, lakes, wood bridges, rivers and caves enjoying the sound and color of nature.

Guided Imagery therapy (IT): Another related method for relief of severe pain is to use the VR system to guide the patient through a series of mental states. This is one of the most effective therapies in the United States for pain relief as explained in a study published by a medical research team led by Wolsko [16]. The process of guided imagery is illustrated in Figure 1B. In a (three-step) Guided Imagery therapy:

- The initial mental state of the patient is one where the patient experiences severe pain.

- By presenting imagery that catches the patient's attention, the system changes the patient's mental state to a next state where the patient experiences only moderate pain.

- Again by presenting imagery that again catches the patient's attention, the system further changes the patient's mental state to a final state where the patient experiences only little or no pain.

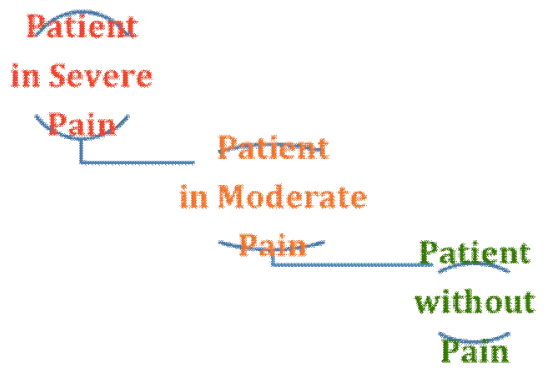

\section{Figure 1B: A Diagram of Mental States of Guided Imagery from High Pain to without Pain}

The process of guided imagery has a long history, and may have used for example by native Americans for reduction of pain prior to the modern era.

As an interesting example of the prior use of Guided Imagery therapy using immersive VR, the co-author and his team developed virtual reality software "Imagery Way" to enhance the immune system and reduce pain [17]. It is designed for women suffering from breast cancer. "Imagery way" immerses patients in the environment to attract their attentions and dives into deep relaxation in the treatment of breast cancer includes course of chemotherapy, radiation therapy or surgery. When chemotherapy is used, different kind of side effects are reflected on patients such as anxiety, nausea, depression and vomiting. Imagery way immerses patients into nature with a natural green environment, sustainable water flow and pleasant bird whispering. Silence and relaxation helps patient to hear the sound of nature, waterfalls, birds and bugs. The movements of insects rendered via virtual reality effects attract the attention of the patient and release its pain. The co-author's "Imagery Way" is thus a clear illustration of the power of VR-guided imagery for pain therapy.

The Challenge: In this Guided Imagery therapy, the system's control of the subject's attention is critical. The next section considers novel ways to increase the system's control of the subject's attention.

\section{Natural and Artificial Synesthesia}

\section{III.1 Natural Synesthesia}

Natural Synesthesia was first recognized in the scientific community in 1893 [18] .[19] provides an overview (also see [20] for their similarity to metaphors). Natural Synesthesia has for the last decade been an important emerging area in brain science, very useful for understanding of the brain's processing of sensory information. Textbooks on Natural Synesthesia include [21], [22],[23], [24], [25], [63], [26], [27], [28], [29], [30], [31] and[32]. 


\section{III.2 Prior Literature on Artificial Synesthesia and Prior Demonstrations}

There is a small but emerging body of prior works on the use of Artificial Synesthesia to enhance VR, for example to color particular objects in a visual scene to enhance the subject's attention to those objects. Prior Demonstrations of Synthetic Synesthesia include:

(a) The Synthetic Synesthesia system developed by the Eagleman Lab (Laboratory for Perception and Action, Departments of Neuroscience and Psychiatry, Baylor College of Medicine, Houston, TX): displays Artificial Synesthesia associations between various senses, including color and sound.

(b) [33] and [34] developed a Synesthetic Music Experience Communicator which provided visual display in response to music to the goal of illustrating the experience of visual Synesthesia in response to musical sound.

(c) Thórisson and Donoghue [35] at the The Media Laboratory, MIT developed a interface to mix distinct sound sources (e.g., the flute, guitar and organ) called the Geometric Sound Mixer (GSM), that displays the sound sources on a 2D plane and allows the user to mix these by selecting positions on the plane between the positions of the sound sources on the 2D plane.

(d) Addison's Project (at CAVE, San Diego Supercomputer Center, CA) is an immersive VR room that provides visual perception of body sensors for breathing, heart rate, etc.

(e) [36], [37], and [38] developed scoreLight which is a musical instrument that generate sound from lasar-scanning of the lines of drawings and from the contours of three-dimensional objects.

(f) Plouznikoff et al [65] demonstrated artificial grapheme-color synesthesia using a wearable display.

Limitations of Prior Demonstrations of Artificial Synesthesia: These prior works in Artificial Synesthesia in VR were mostly motivated by a desire to allow the subject experience Natural Synesthesia, rather than improve the performance or control the attention of the subject.

\section{III.3 Novel Use of Artificial Synesthesia for Pain Therapy}

There appears to be little or no prior literature on use of artificial synesthesia for pain therapy. Since artificial synesthesia has the effect of combining sensory stimuli from distinct senses, it appears very likely be effective in enhancing the control of a person's attention. Therefore the use of artificial synesthesia in VR has the potential of improving and directing sensory distraction to provide improved pain reduction. It is not reasonable to expect that application of VR-trained artificial synesthesia attention control in VR allows us to completely switch ON/OFF the pain sensation, but expect it to reduce the perceived pain in a degree measurable.

III.4 Exploiting the Underlying Structure of Sensory Perception to Further Control \& Direct the Subject's Attention Our work in Artificial Synesthesia in VR is also exploiting the underlying structure of the senses involved, to further capture the attention of the subject and so better control and direct the subject's attention.

Each of the various senses such as color, character visual processing, sound pitch and spatial tactile sensations appear to have an underlying structure, modeled in prior works by a spatial representation (a continuous $2 \mathrm{D}$ maps) which we call a sense-map.

(a) For example, colors can be arranged in perceived similarity around a wheel to form a color-map (this idea dates to Newton's book Optics [39]), whose angular position is color frequency, and radial coordinate provides for hue intensity. This was later refined by an artist Munsell, who provided a geometric model (which is two dimensional if grey value tones are not modeled) for hue spacing from judgments of relative color similarity, such as illustrated below:

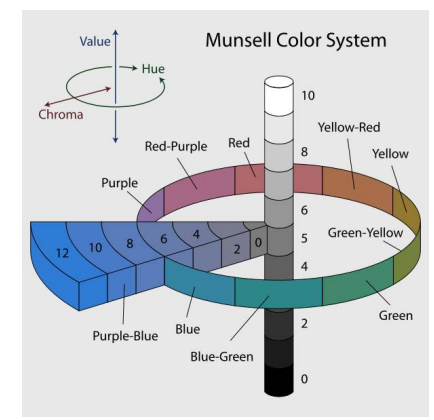

Figure 2 illustration of Munsell Hue spacing from judgments of similarity (from http://en.wikipedia.org/wiki/File:Munsell-system.svg)

(b) As a further example, characters can be arranged in spatial maps where proximity implies similar geometric properties. (c) Also, tones can be arranged in an array (a tone-map) that reflects similarity of frequency as well as tonal patterns of groups of sounds such as chords, as investigated by Dmitri Tymoczko [40] in his work on the geometry of musical chords. 


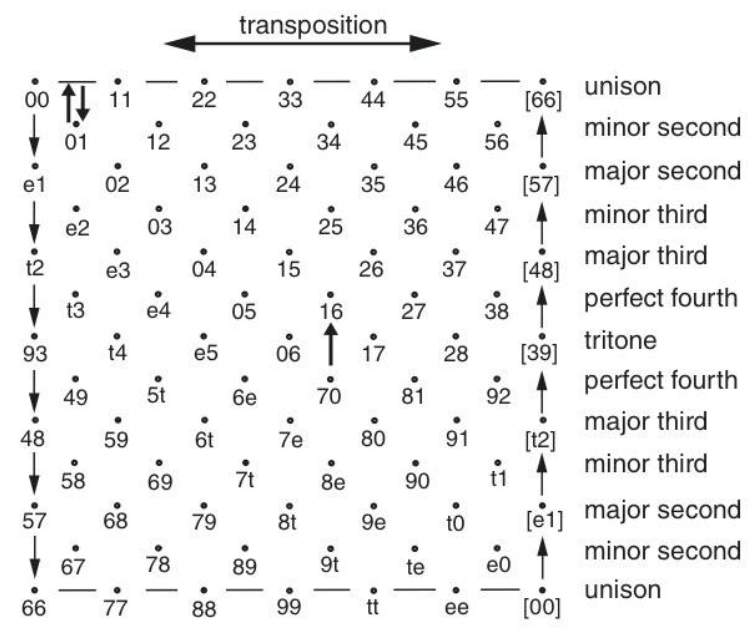

Figure 3: Tymoczko's 2D diagram of Tones and Chords (from Figure 2, page 73 of [40] Tymoczko, Dmitri, The Geometry of Musical Chords, Science, 7 July 2006, Vol. 313, p 72-74.)

(d) Somatotopy is a one-to-one correspondence of an area of the body to a specific position on the brain. The sensory homunculus [65] (an idea due to Wilder Penfield) is a nonuniform 2D map of this somatotopy, describing how the tactile and other sensory information from parts of the body are mapped to corresponding cortical sites in the brain. Considerable prior research has been done on the mechanisms for Natural Synesthesia involving auditory-tactile somatosensory associations [41], [42], including feeling sounds after thalamic lesions [43], [44] and other multisensory interplay between "sensory-specific" brain regions [45], [46].

By underlying structure of the senses involved, to further capture the attention of the subject and so better control and direct the subject's attention.

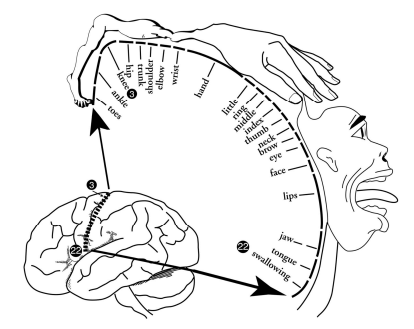

Figure 4: Penfield's Illustration of a Homunculus - a $\quad$ somatotopic brain map (From http://www.intropsych.com/ch02_human_nervous_system/homunculus.html)

We are working to enhance immersive VR by first known use of what we call Structured Artificial Synesthesia, where the coupling between senses also couples the structure of the various senses by a mapping between the corresponding sense-maps. To do this, we choose mappings between sense-maps of distinct senses (for example transfer of pleasant harmonic chords to pleasantly perceived groupings of another sense such as of colors).

\section{III.5 Possible Use of Neural Plasticity}

Recall that neural plasticity is a general term for the ability of the Brain to change dynamically, as discussed in section 1.5 . In the last decades it was shown that neural plasticity could also occur in mature, uninjured individuals by various types of training or external stimuli. Perhaps the most dramatic demonstrations of neural plasticity has been the work of Vilayanur Ramachandran, who [24] made experiments which made ingenious use of mirrors in demonstrating the neural plasticity of these displaced somatotopic brain maps. In particular, for amputees with perception (this is known as a phantom limb) of the missing arm, he used an arrangement of mirrors to mirror the visual perception of the movement of the existing arm with a perceived identical movement of the missing arm, inducing a change in the patient's somatotopic brain map to decrease lost 
limb pain. Subsequent investigations by [48], [49], [65], [50] have extended this work. Also, [51] demonstrated physiologically impossible movement of their amputees' phantom limbs. [52] demonstrated motor control training for uninjured mature individuals. Applications of neural plasticity to brain-machine interfaces have been discussed [53], [54], and [55] demonstrated adaptive training of control of actuation across a brain-machine interface. [56] demonstrated the use of training as therapies for elder adults.

Potentially, our work in Artificial Synesthesia using VR could also also exploit neural plasticity, by adaptive training of the attention (e.g., direction of the eyes) of subject to particular types of movement of a particular captivating object in view; this training could improve the control and direction of the subject's attention.

\section{Development and Applications of a VR system that introduces Artificial Synesthesia associations}

\section{IV.1. A Prototype VR System for Demonstration of Artificial Synesthesia}

Recall a grapheme is a character, symbol or number. Graphemes are generally far easier to introduce or detect in a graphic scene than general objects, and so are used in our prototype system rather than general graphic objects.

We are developing a VR system that introduces Artificial Synesthesia associations in the VR system's (visual, sound, and tactile) output associated with specific graphemes. These Artificial Synesthesia associations include:

- coloring specific graphemes with specific colors

- coloring specific graphemes where the specific graphemes emit specific sounds

- producing specific sounds in association with specific graphemes

- producing specific tactile sensations in response to specific graphemes

- producing specific tactile sensations in response to specific colors

This work involves insertion and/or detection of specific graphenes, colors, or sounds, and insertion of the associated specific graphene, color, sound, or tactile sensation.

After achieving training of pair-wise Artificial Synesthesia, we are also attempting to train triplets of associations between three distinct senses (triplet Artificial Synesthesia).

\section{IV.2. Investigation of Structured Artificial Synesthesia}

Recall, as described in detail in our introduction, that sense-maps are continuous 2D maps of the senses such as color, character visual processing, sound pitch and spatial tactile sensations.

We are exploiting this rich underlying structure of the senses, to investigate Structured Artificial Synesthesia, where the coupling between senses also couples the structure of the various senses by a mapping between the corresponding sense-maps. A key challenge is determine how to map between two sensory classes (visual graphene, sound, tactile) is such a way as to make use of the known sense-map of these sensory classes. To do this, we choose mappings between sense-maps of distinct senses, and test what mappings provide for better performance.

We are using two approaches:

(1) It is known that individual senses such as sound (or color) have pairings and groupings that are naturally harmonious these are for example chords - or can be to various degrees inharmonious. These groupings have been spatially mapped on 2 dimensional charts. We are experimenting the transfer of pleasant harmonic chords to pleasantly perceived groupings of another sense such as a set of "harmonious" colors.

(2) Further, we are utilizing and further refining network models of each of the distinct sense-maps (e.g., Munsell Hue spacing as a simple network model for colors and Tymoczko's 2D diagram of Tones and Chords as a simple network model for sounds). Then we determine mappings between these of the distinct sense-maps that preserve locality or other properties such as complementarity within each of the sensory class that we wish to associate via Artificial Synesthesia.

\section{IV.3. Tests and Applications of our VR System for Artificial Synesthesia:}

A key challenge here is to develop performance metrics that can be rigorously tested. These performance metrics which we are developing are related to the degree the subject has enhanced response to the introduction of graphemes, colors, sounds and/or tactile simulations, when these are augmented with the Artificial Synesthesia sensory stimuli.

A further challenge is to test and verify (using rigorous statistical methods as used in experimental psychology), that VR system that introduces Artificial Synesthesia associations actually provided improved performance in the metrics we define. Here we are intending to apply techniques already developed in experimental psychology, for example for demonstrating Natural Synesthesia associations between specific colors and sounds. between specific colors and tactile sensations [57]. For example, to test whether we have successfully introduces Artificial Synesthesia associations between specific pairs of colors and graphemes, we plan to apply the technique developed by Vilayanur Ramachandran in his work on verifying existing Natural Synesthesia associations between specific pairs of colors and graphemes (e.g., visual perceived characters and numbers), by showing that color/character pairing results in much faster recognition of spatial patterns of graphemes which have synesthesicia pairings with distinctive colors. (also see [58], [59], [60], [61], [62]). 


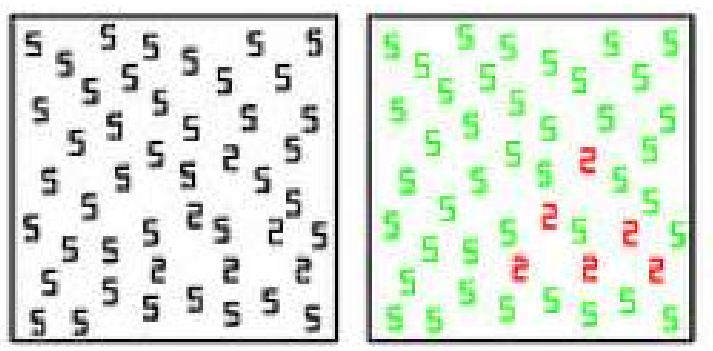

Figure 5: Spatial Patterns of Graphemes asassociated by Color: If the character 2 is associated with the color red, then the triangle of $2 \mathrm{~s}$ in the right figure is quickly recognized by detection of the red triangle shape (from [58] Hubbard EM, Arman AC, Ramachandran VS, Boynton GM (March 2005). "Individual differences among grapheme-color synesthetes: brain-behavior correlations" Neuron 45 (6): 975-85. doi:10.1016/j.neuron.2005.02.008)

\section{IV.4. Application of our VR System for Artificial Synesthesia to influence pain perception:}

As described in more detail in section II, Alhalabi and number of other investigators have previously used VR to distract patients from pain and/or discomfort, and demonstrated considerable success in this task. We are planning to test applications of our VR System for Artificial Synesthesia to influence pain perception.

IV.4. Inducing Subliminal Synesthesia: A by-product of our main work on Immersive VR) is to induce via Immersive VR a subliminal form of Synesthesia that is not perceived by the subject but provides enhanced ability at various perception tasks. To this end, we are attempting to induce via training a Natural Synesthesia that is not consciously perceived by a subject.

Training of induced Subliminal Synesthesia can be used to probe the transfer of underlying structure of distinct senses. In particular, these VR trained induced synesthesia associations may also induce a mapping between the structural organizations involving these senses.

\section{Conclusions}

This paper provided an interdisciplinary study of novel applications of techniques and tools of an emerging topic of brain science known as Synesthesia to the area of computer science generally known as Immersive Virtual Reality, that makes the subject's awareness of physical self be diminished by being surrounded in an engrossing artificial environment.

Synesthesia is associations between distinct senses, e.g., perception of particular colors associated with particular graphemes, sounds, tactile sensations, taste or smells. In Natural Synesthesia, the sensory associations that the subject perceives appear to be real perceptions, but are actually created within the brain, whereas in Artificial Synesthesia induced associations between senses created synthetically, for example via VR, rather than created by the subjects brain. While Natural Synesthesia is present in only a small proportion of the population, potentially Artificial Synesthesia can be experienced by anyone using a VR system. Natural Synesthesia has for the last decade been an important emerging area in brain science; we feel Artificial Synesthesia could have similarly important applications to VR. The paper describes the enhancement of immersive VR by use of Artificial Synesthesia to improve the system's performance at steering and directing the attention of the user. We describe techniques for an enhanced Immersive VR that displays associations between a variety of senses: between colors and characters, also between colors and sounds, and between sounds and the position of tactile sensations. The sense association provided by Artificial Synesthesia allows the system to better capture the user's attention and better direct that attention.

Our paper mentions that a small but emerging body of prior works on the use of Artificial Synesthesia to enhance VR, in particular to color particular objects in a visual scene to enhance the subject's attention to those objects. However, this prior works in Artificial Synesthesia in VR has been limited mostly to coloring objects, and more over has not exploited the underlying structure of the senses involved. A further innovative topic described by this paper is to also exploit the rich underlying structure of the senses. The various senses such as color, character visual processing, sound pitch and spatial tactile sensations each are known to have an underlying complex structure, generally modeled in prior works by a spatial representation (a continuous 2D map) which we term a sense-map (e.g., color maps for visual perception, tone and chord maps for sounds, etc.). We described how we may further enhance immersive VR by the first known use of what we call Structured Artificial Synesthesia, where the coupling between senses also couples the structure of the various senses by a mapping between the corresponding sense-maps. To do this, we described the technique of choosing mappings between sense-maps of distinct senses (for example transfer of a pleasant harmonic chords to pleasantly perceived groupings of another sense such as of colors).

We discussed a very high-impact medical application of this basic research in Artificial Synesthesia to medical science: pain relief. Immersive VR has been extensively applied for pain therapies, by using VR to distract the patient from painful sensations and redirect attention to mental states associated with less pain (this often involves techniques of guided imagery, 
where the attention of patient is directed through a series of mental states where the patient experiences progressively less pain). In spite of the power of these pain distraction and guided imagery techniques, a critical challenge is to control and redirect the attention of the patient. We argued that a major application of this work in VR-induced Artificial Synesthesia is to provide an enhanced methodology for controlling the attention of the subject, and to improve the direction of attention of subjects undergoing guided imagery pain therapies, so providing improved methods for directing the attention of subjects away from pain. Other potential high-impact applications of this research include improved immersive VR, more programmable human/computer interfaces and other medical therapies.

\section{Acknowledgements}

We wish to acknowledge support to both coauthors by King Abdulaziz University (KAU) as well as support to John H Reif NSF CCF-1141847, NSF CCF- 1217457, and NSF CCF-1320360.

\section{REFERENCES}

Rheingold, Howard Virtual Reality.Summit, (1991). ISBN 0-262-68121-8.

Pimentel, K., \& Teixeira, K. (1993). Virtual reality. New York: McGraw-Hill. ISBN 978-0-8306-4065-2

[1] W.S. Al-Halabi, S.A. Aseeri, R.A. Almeleak, and H.T. Al-Hashmie "Effect of Virtual Reality on Breast Cancer Patients" In the Proceedings of the Modeling and Simulation conference in Quebec City, Canada 26-28/5/2008

[2] Hoffman H.G., Patterson D.R., Carrougher G.J., \& Sharar S.(2001). The Effectiveness of Virtual Reality Based Pain Control with Multiple Treatments, Clinical Journal of Pain, 17, 229-235

[3] Schneider S.M., (2003), Virtual Reality as a Distraction Intervention for Women Receiving Chemotherapy, CyberPsychology \& Behavior ,Jun 2003, Vol. 6, No. $3: 301-307$.

[4] Andreas A.J. Wismeijer, Ad J.J.M. Vingerhoets, The Use of Virtual Reality and Audiovisual Eyeglass Systems as Adjunct Analgesic Techniques: A Review of the Literature, Annals of Behavioral Medicine, 30, 2005, 268.

[5] Sullivan C, Schneider PE, Musselman RJ, Dummett CO Jr, Gardiner D, The effect of virtual reality during dental treatment on child anxiety and behavior, ASDC J Dent Child, 67(3), 2000, 193-6, 160-1.

[6] Schneider S., Workman M.L., (2000), Virtual Reality as a Distraction Intervention for Older Children Receiving Chemotherapy. Pediatric Nursing, Nov-Dec, 2000: 593-597

[7] Hoffman H. G.,Azucena G., Patterson D.R., , Jensen M., Furness T., William F. A., (2001), The Effectiveness of Virtual Reality for Dental Pain Control: A Case Study, CyberPsychology \& Behavior. August 1, 2001, 4(4): 527-535. doi:10.1089/109493101750527088. (b)

[8] W.S. Al-Halabi, A. Alsalmi, "The Impact of Imagery Therapy on Rheumatoid Arthritis Treatments" In the Proceedings of International Conference on Modelling, Identification and Control, MIC 2009, Innsbruck, Austria , February 16 -18, 2009

[9] W. S. Al-Halabi, A. Alsubhi, A. Alrajhi, M. Alqurashi,"Using Virtual Reality to Cure Paralysis Caused by Brain Stroke” In the proceedings of the 3rd International conference on disability \& rehabilitation, Riyadh, KSA, March 22-26, 2009

[10] W. S. Al-Halabi, A. Alsubhi Use of Computer Generated Environments to Cure Post-Stroke Paralysis" Accepted for publishing in International Conference on Modeling and Simulation, Banff, Alberta, Canada, July 6-8, 2009

[11] W. Alhalabi and M. Al-Qurashi "Imagery Therapy for Pain Control Using Computer Generated Virtual Reality" Frontiers in Neuroengineering, Annual CyberTherapy and CyberPsychology 2009 conference. doi: 10.3389/conf.neuro. 14.2009.06.004

[12] F.Y. Al-Zamzami and W.S. Al-Halabi, "Using Guided Imagery as a Distraction Method during Dental Procedures" In the Proceedings of the Modeling and Simulation conference in Quebec City, Canada 26-28/5/2008

[13] W. Alhalabi, S Aseeri, "The Impact of Virtual Reality and Computer Game on Imagery Therapy for Cancer Patients" In the Proceedings of the Seventh Saudi Engineering Conference, Riyadh, 2-5/12/2007

[14] W. Alhalabi, R Al-meleakm, "Effect of Virtual Reality on Breast Cancer Patients" In the Proceedings of the Seventh Saudi Engineering Conference, Riyadh, 2-5/12/2007

[15] W. S. Al-Halabi, The Impact of Imagery Therapy on Rheumatoid Arthritis Patients (2012). International Journal of Computer Science Issues, Vol 9, No 5

[16] Wolsko, P. Davis R., Eisenberg D., Phillips R \& (2004). Use of Mind-Body Medical Therapies Results of a National Survey. Journal of General Internal Medicine, Vol 19, No 1, pp. (43-50), ISSN 0884-8734

[17] Alhalabi, W., Aseeri S., Almeleak R., Alhashmie H., (2008), Effect of Virtual Reality on breast cancer patients Proceedings of the 19th IASTED International Conference on Modelling and Simulation.

[18] Jain, Neeraj; Qi, HX; Collins, CE; Kaas, JH (October 22, 2008). "Large-Scale Reorganization in the Somatosensory Cortex and Thalamus after Sensory Loss in Macaque Monkeys", The Journal of Neuroscience 28 (43): 11042-11060. doi:10.1523/JNEUROSCI.2334-08.2008. PMC 2613515.

[19] Bhuvana Balasubramanian, Wendy Portillo, Andrea Reyna, Jian Zhong Chen, Anthony N. Moore, Pramod K. Dash, and Shaila K. Mani, (2008). Nonclassical Mechanisms of Progesterone Action in the Brain: I. Protein Kinase C Activation in the Hypothalamus of Female Rats, Endocrinology 149(11):pages 5509. doi: 10.1210/en.2008-0712

[20] Michael J. Higley and Stephen M. Strittmatter, (2010). Lynx for Braking Plasticity, Science 330, 1189-1190. DOI: 10.1126/science.1198983.

[21] Cutler, Sarah M.; Hoffman, Stuart W.; Pettus, Edward H.; Stein, Donald G. (October 2005)."Tapered progesterone withdrawal enhances behavioral and molecular recovery after traumatic brain injury". Experimental Neurology (Elsevier) 195 (2): 423-429. doi:10.1016/j.expneurol.2005.06.003. PMID 16039652.. 
[22] Cutler, Sarah M.; Cekic, Milos; Miller, Darren M.; Wali, Bushra; VanLandingham, Jacob W.; Stein, Donald G. (September 24, 2007). "Progesterone Improves Acute Recovery after Traumatic Brain Injury in the Aged Rats". Journal of Neurotrauma 24 (9): 1475-1486. doi:10.1089/neu.2007.0294. PMID 17892409.

[23] Ramachandran, Vilayanur S.; Hirstein, William (1998). "The perception of phantom limbs. The D. O. Hebb lecture", Brain 121 (9): $1603-1630$. doi: 10.1093/brain /121.9.1603. PMID 9762952.

[24] Ramachandran VS, Rogers-Ramachandran D (April 1996). Synesthesia in phantom limbs induced with mirrors. Proc. Biol. Sci. 263 (1369): $377-86$. doi:10.1098/rspb.1996.0058. PMID 8637922.

[25] Richard E. Cytowic and David M. Eagleman, Wednesday Is Indigo Blue: Discovering the Brain of Synesthesia, The MIT Press, 2009

[26] Dann, K. (1998). Bright Colors Falsely Seen. Cambridge: Harvard University Press. ISBN 0-300-06619-8.

[27] Duffy, P. L. (2001). Blue Cats and Chartreuse Kittens: How Synesthetes Color their Worlds. New York: Henry Holt \& Company. ISBN 0-7167-40885.

[28] Harrison, J. (2001). Synesthesia: The Strangest Thing. Oxford: Oxford University Press. ISBN 0-19-263245-0.

[29] V.S. Ramachandran, M.D., Ph.D., and Sandra Blakeslee, Phantoms in the Brain: Probing the Mysteries of the Human Mind, (1998)

[30] Robertson, L. and Sagiv, N. (Eds., 2005). Synesthesia: Perspectives from Cognitive Neuroscience. Oxford: Oxford University Press. ISBN 0-19516623-X.

[31] Sagiv, Noam; Robertson, Lynn C (2005). Synesthesia: perspectives from cognitive neuroscience. Oxford: Oxford University Press. ISBN 0-19516623-X. OCLC 53020292

[32] Ward, J. (2008) The Frog who croaked Blue: Synesthesia and the Mixing of the Senses. Routledge. ISBN 978-0-415-43014-2

[33] Lewis Hill and James Oliver, Development of a Synesthetic Music Experience Communicator, www.elchill.com/smec/smec_hill_oliver.pdf

[34] Lewis Charles Hill II, Synesthetic music experience communicator. PhD thesis, Iowa State University, (2006).

[35] Thórisson, K. R. \& Donoghue, K. Synthetic synesthesia: Mixing Sound with Color, InterChi Adjunct Proceedings, 65-66, (1993).

[36] A. Cassinelli, Y. Kuribara, D. Manabe and M. Ishikawa, scoreLight, Digital Content Expo 2009 Symposium (25 October 2009, Miraikan - Museum of Emerging Science and Innovation, Tokyo)

[37] A. Cassinelli, Y. Kuribara, D. Manabe and M. Ishikawa: scoreLight: a laser-based synesthetic experience, additional documentation for SIGGRAPH ASIA 2009 (Art Gallery).

[38] A. Cassinelli, Y. Kuribara, A. Zerroug, D. Manabe and M. Ishikawa, scoreLight: playing with a human sized laser pickup, International Conference on New Instruments for Musical Expression (NIME2010), 15-18th June 2010 Sydney, Australia, pp:144-149, (2010).

[39] Newton, Isaac (1704). Opticks or, a treatise of the reflexions, refractions, inflexions and colours of light : also two treatises of the species and magnitude of curvilinear figures.

[40] Tymoczko, Dmitri, The Geometry of Musical Chords, Science, 7 July 2006, Vol. 313, p 72-74.

[41] Foxe JJ, Wylie GR, Martinez R, Schroeder CE, Javitt DC, Guilfoyle D, Ritter W, Murray MM. (2002) Auditory-somatosensory multisensory processing in auditory association cortex: an fMRI study. J Neurophysiol 88: 540-543.

[42] Keysers C, Wicker B, Gazzola V, Anton J-L, Fogassi L, Gallese V. (2004) A touching sight: SII/PV activation during the observation and experience of touch. Neuron 42: 335-346.

[43] Ro T, Farne` A, Johnson RM, Wedeen V, Chu Z, Wang ZJ, Hunter JV, Beauchamp MS. Feeling sounds after a thalamic lesion. Ann Neurol 62: 433441, 2007.

[44] Beauchamp MS, Ro T. (2008) Neural substrates of sound-touch synesthesia after a thalamic lesion. J Neurosci 28: $13696-13702$.

[45] Driver J, Noesselt T. (2008) Multisensory interplay reveals crossmodal influences on "sensory-specific" brain regions, neural responses, and judgments. Neuron 55: 11-23.

[46] Cappe C, Morel A, Barone P, Rouiller EM. (January 15, 2009). The thalamocortical projection systems in primate: an anatomical support for multisensory and sensorimotor interplay. Cereb Cortex doi:10.1093/cercor/bhn228.

[47] Flor H, Elbert T, Knecht S, Wienbruch C, Pantev C, Birbaumer N, et al. Phantom-limb pain as a perceptual correlate of cortical reorganization following arm amputation. Nature 1995; 375: 482-484.

[48] Flor, H. (July 2002). "Phantom-limb pain: characteristics, causes, and treatment". The Lancet Neurology (Elsevier) 1 (3): 182-189. doi:10.1016/S1474$4422(02) 00074$.

[49] Flor H, Cortical Reorganization And Chronic Pain: Implications For Rehabilitation, J Rehabil Med, 2003, Suppl.41:66-72.

[50] McCabe CS, Haigh RC, Blake DR (April 2008). "Mirror visual feedback for the treatment of complex regional pain syndrome (type 1)". Curr Pain Headache Rep 12 (2): 103-7. doi:10.1007/s11916-008-0020-7 PMID 18474189

[51] Moseley, Brugger, Interdependence of movement and anatomy persists when amputees learn a physiologically impossible movement of their phantom limb, PNAS, Sept 16, 2009.

[52] Liu, Yu-Fan; Chen, Hsuin-ing; Wul, Chao-Liang; Kuol, Yu-Min; Yu, Lung; Huang, A-Min; Wu, Fong-Sen; Chuang, Jih-Ing; and Jen, Chauying J. (2009). "Differential effects of treadmill running and wheel running on spatial or aversive learning and memory: Roles of amygdalar brain-derived neurotrophic factor and synaptotagmin I." Journal of Physiology 587(13): 3221-3231. doi:10.1113/jphysiol.2009.173088

[53] Donoghue, John P. (2002). "Connecting cortex to machines: recent advances in brain interfaces", (2002). Nature Neuroscience 5: $1085-1088$. doi:10.1038/nn947. PMID 12403992.

[54] Lebedev, Mikhail A.; Carmena, Jose M.; O'Doherty, Joseph E.; Zacksenhouse, Miriam; Henriquez, Craig S.; Principe, Jose C.; Nicolelis, Miguel A. L. (May 11, 2005). "Cortical Ensemble Adaptation to Represent Velocity of an Artificial Actuator Controlled by a Brain- Machine Interface" The Journal of Neuroscience 25 (19): 4681-4693. doi:10.1523/JNEUROSCI.4088-04.2005. PMID 15888644.

[55] Cohen, Wendy; Hodson, Ann; O'Hare, Anne; Boyle, James; Durrani, Tariq; McCartney, Elspeth; Mattey, Mike; Naftalin, Lionel et al. (June 2005). "Effects of Computer-Based Intervention Through Acoustically Modified Speech (Fast ForWord) in Severe Mixed Receptive-Expressive Language Impairment: Outcomes From a Randomized Controlled Trial". Journal of Speech, Language, and Hearing Research 48: 715-729. doi:10.1044/10924388(2005/049). 
[56] Mahncke, Henry W.; Connor, Bonnie B.; Appelman, Jed; Ahsanuddin, Omar N.; Hardy, Joseph L.; Wood, Richard A.; Joyce, Nicholas M.; Boniske, Tania et al. (August 15, 2006). "Memory enhancement in healthy older adults using a brain plasticity-based training program: a randomized, controlled study", Proceedings of the National Academy of Sciences of the USA 103 (33): 12523-12528. doi:10.1073/pnas.0605194103 PMC 1526649. PMID 16888038 .

[57] Ramachandran VS, Brang D. (2008). "Tactile-emotion synesthesia". Neurocase 14 (5): 390-399. doi:10.1080/13554790802363746 PMID 18821168.

[58] Hubbard EM, Arman AC, Ramachandran VS, Boynton GM (March 2005). "Individual differences among grapheme-color synesthetes: brain-behavior correlations" Neuron 45 (6): 975-85. doi:10.1016/j.neuron.2005.02.008

[59] Hubbard EM, Ramachandran VS (2005). "Neurocognitive mechanisms of synesthesia". Neuron 48 (3): 509-520. doi:10.1016/j.neuron.2005.10.012. PMID 16269367.

[60] Brang D, Hubbard EM, Coulson S, Huang M, Ramachandran VS (2010). "Magnetoencephalography reveals early activation of V4 in grapheme-color synesthesia". Neuroimage 53 (1): 268-274. doi:10.1016/j.neuroimage.2010.06.00. PMID 20547226

[61] Brang D, Teuscher U, Ramachandran VS, Coulson S.(2010). "Temporal sequences, synesthetic mappings, and cultural biases: the geography of time". Consciousness and Cognition 19 (1): 311-320. doi:10.1016/j.concog.2010.01.003. PMID 20117949.

[62] Teuscher U, Brang D, Ramachandran VS, Coulson S. (2010). "Spatial cueing in time-space synesthetes: An event-related brain potential study". Brain and Cognition 74 (1): 35-46. doi:10.1016/j.bandc.2010.06.001 PMID 20637536.

[63] Cytowic, R.E. \& Eagleman, D.M. (2009) Wednesday is Indigo Blue: Discovering the Brain of Synesthesia, with an afterword by Dmitri Nabokov. Cambridge: MIT Press ISBN 978-0-26-201279-9.

[64] Plouznikoff, N., Plouznikoff, A., Robert, J.: Artificial Grapheme-Color Synesthesia for Wearable Task Support. In . Proceedings. of the Ninth IEEE International Symposium on Wearable Computers. ISWC(2005), Oct. 18-21 , 2005, pp. 108 - 111 (ISBN: 0-7695-2419-2 In 108-113).

[65] Schott GD. Penfield's homunculus: a note on cerebral cartography. J Neurol Neurosurg Psychiatry. 1993 Apr;56(4):329-333. 